\title{
Review of: "Metrics based on information entropy applied to evaluate complexity of landscape patterns"
}

\author{
EDSON SANO
}

Potential competing interests: The author(s) declared that no potential competing interests exist.

In this study, the authors created two scripts named CompPlex HeROI and ComPlex Janus to estimate and map entropy (He), variability (He/Hmax), López-Ruiz, Mancini, and Calbet (LMC) and Shiner, Davison, and Landsberg (SDL) measures. The methodological approach and data analysis are fine; however, I have the following comments:

1. Title. The title should emphasize the creation of two scripts, which is the basis of the paper.

2. Abstract. It is impossible to have any idea of what are the meanings of López-Ruiz, Mancin, and Calbet (LMC) and Shiner, Davison, and Landsberg (SDL) measures if the readers are not aware of related publications. Besides, the authors do not provide any values of entropy, variability, LMC, and SDL measurements found in the study area and their main conclusions regarding the spatial complexity of the landscape. The readers even do not know if the study area presents low, intermediate, or high complexity. 3. Study area. What is the difference between ecological and experimental parks? The boundaries of the Figures 1 and 7 do not match.

4. Methods. The authors stated that Shiner et al. (1999) classified complexity measures based on information entropy into three broad categories 1, 2, and 3. Then, the authors selected categories 1 (He and $\mathrm{He} / \mathrm{Hmax}$ ) and 3 (LMC and SDL) to evaluate the spatial complexity of the study area. I do not know if I have missed any important information here, but the question I raise here is what were the criteria to select these two categories? In another words, why the authors do not assessed the category 2 ?

5. Regions of interest (ROIs). My major objection of the paper is here. How did the authors decide about the location, size, and shape of the ROIs? Why only one polygon per class? I believe the authors could run some image segmentation and then select the ROls from this step. The ultimate question here is if the results will be similar if we select another set of training samples.

6. Land use classes. Not all spectral classes analyzed in this study are land use classes. Native vegetation classes are land cover classes, not land use classes. The majority of remote sensing papers use the terminology "land use and land cover", "land use/cover" or "land cover and land use".

7. The scripts. The use of only one single spectral band to run the two scripts seems to be a serious limitation. I do not know if there is any programming limitation, but the scripts should be able to consider multispectral bands to produce and map He, He/Hmax, SDL, and LMC metrics.

8. Conclusions. There are too many citations in the Conclusions section, which is more likely discussion 
than conclusion. 